\title{
A test of Gaia Data Release 1 parallaxes: implications for the local distance scale
}

\author{
Stefano Casertano ${ }^{1,2}$, Adam G. Riess ${ }^{2,1}$, Beatrice Bucciarelli ${ }^{3}$, and Mario G. Lattanzi ${ }^{3}$ \\ 1 Space Telescope Science Institute, 3700 San Martin Drive, Baltimore, MD 21218, USA \\ e-mail: stefano@stsci.edu \\ 2 Department of Physics and Astronomy, Johns Hopkins University, Baltimore, MD 21218, USA \\ 3 INAF, Osservatorio Astronomico di Torino, Strada Osservatorio 20, 10025 Pino Torinese, TO, Italy
}

Received 16 September 2016 / Accepted 23 November 2016

\begin{abstract}
Aims. We present a comparison of Gaia Data Release 1 (DR1) parallaxes with photometric parallaxes for a sample of 212 Galactic Cepheids at a median distance of $2 \mathrm{kpc}$, and explore their implications on the distance scale and the local value of the Hubble constant $H_{0}$.

Methods. The Cepheid distances are estimated from a recent calibration of the near-infrared period-luminosity $(P-L)$ relation. The comparison is carried out in parallax space, where the DR1 parallax errors, with a median value of half the median parallax, are expected to be well-behaved.

Results. With the exception of one outlier, the DR1 parallaxes are in very good global agreement with the predictions from a wellestablished $P-L$ relation, with a possible indication that the published errors may be conservatively overestimated by about $20 \%$. This confirms that the quality of DR1 parallaxes for the Cepheids in our sample is well within their stated errors. We find that the parallaxes of 9 Cepheids brighter than $G=6$ may be systematically underestimated. If interpreted as an independent calibration of the Cepheid luminosities and assumed to be otherwise free of systematic uncertainties, DR1 parallaxes are in very good agreement (within $0.3 \%$ ) with the current estimate of the local Hubble constant, and in conflict at the level of $2.5 \sigma(3.5 \sigma$ if the errors are scaled) with the value inferred from Planck cosmic microwave background data used in conjunction with $\Lambda \mathrm{CDM}$. We also test for a zeropoint error in Gaia parallaxes and find none to a precision of $\sim 20 \mu$ as. We caution however that with this early release, the complete systematic properties of the measurements may not be fully understood at the statistical level of the Cepheid sample mean, a level an order of magnitude below the individual uncertainties. The early results from DR1 demonstrate again the enormous impact that the full mission will likely have on fundamental questions in astrophysics and cosmology.
\end{abstract}

Key words. parallaxes - distance scale - cosmology: observations - stars: variables: Cepheids - space vehicles: instruments

\section{Introduction}

The Gaia mission (Prusti 2012; Gaia Collaboration 2016b), launched in December 2013 by the European Space Agency, will revolutionize our knowledge of individual stellar objects and of the structure of the Milky Way (MW) by providing distance and velocity measurements of unprecedented precision for over a billion individual objects within the MW. Gaia end-ofmission results will also have a major impact on cosmology, enabling the determination of the Hubble constant $H_{0}$ to better than $1 \%$, assuming concomitant control of statistical and systematic errors beyond the parallax measurements (Riess et al. 2016, hereafter R16). The mission is expected to last at least 5 years, and the final astrometric measurements, with expected accuracy $\sim 5-10 \mu$ as for the best-measured stars, will be released in 2022.

The Gaia DR1 (Gaia Collaboration 2016a), which took place September 14, 2016, is based on measurements taken within the first 14 months of observations (Lindegren et al. 2016), and includes data for over a billion stars brighter than magnitude 20.7. Due to the short observing period and the limited number of separate measurements for each target, parallax and proper motion are partially degenerate, and for a majority of the stars only positions are available in DR1. However, for about 2 million stars in common with the
HIPPARCos (Perryman et al. 1997; van Leeuwen 2007) and Tycho-2 (Høg et al. 2000) catalogs, parallaxes and proper motions could be determined as part of the Tycho-Gaia astrometric solution (TGAS; Michalik et al. 2015) and are included in DR1. These stars are typically brighter than visual magnitude 11.5 and have typical parallax uncertainties of $300 \mu$ as.

One of the early difficulties encountered by the Gaia mission has been a somewhat unexpected variation of the Basic Angle, the angular separation between the two fields of view, with an amplitude of several mas, as reported by the on-board Basic Angle Monitor (BAM). Data taken during commissioning (Mora et al. 2014) prove that the variation reported by the BAM is real. Self-calibration procedures have been devised to correct for the Basic Angle variation, and it is expected that the residuals are degenerate with a global zero point error in the parallax (Michalik \& Lindegren 2016). A sample of 135000 AGN has been used as part of the solution and its verification; from an analysis of parallaxes for both HIPPARCOS stars and AGN, Lindegren et al. (2016) suggest that parallax zero point errors are $\lesssim 0.1$ mas in magnitude, with possible zonal variations and color terms, and a systematic difference between northern and southern hemispheres.

Lindegren et al. (2016) offer several observational and scientific tests and validations of the TGAS results. Because of 
the inherent challenge in reaching a new level of parallax precision, it is important to produce additional tests of the set of Gaia parallax measurements. Here we employ an additional validation, based on the sample of 249 Galactic Cepheids in van Leeuwen et al. (2007), at a median distance of $2 \mathrm{kpc}$. A similar test, based on the sample of Cepheids in Fouqué et al. (2007) but excluding nearby Cepheids (within $1 \mathrm{kpc}$ ), is reported in Sect. C.3 of Lindegren et al. (2016). Our analysis is comparable to theirs, and leads to similar results. However, we use a more recent Cepheid calibration (R16) with well-understood uncertainties, and we are able to obtain a quantitative assessment of the reported DR1 errors, and to derive interesting, albeit tentative, implications on $H_{0}$. We also find some evidence for a modest parallax underestimate for the brightest and nearest Cepheids, which are explicitly excluded from the Lindegren et al. (2016) analysis.

The Cepheid $P-L$ relation, called the Leavitt Law (Leavitt \& Pickering 1912), provides a tight correlation between period and luminosity of Fundamental Mode Cepheids and has been central to the determination of the scale of the Universe for a century. Yet most Galactic Cepheids are at a distance of a few kpc; until recently, this placed them well beyond the useful range for measurements of accurate trigonometric parallaxes. Benedict et al. $(2002,2007)$ used the Fine Guidance Sensors (FGS) on the Hubble Space Telescope (HST) to measure the parallaxes of ten Cepheids within $0.5 \mathrm{kpc}$, reaching individual precisions of 150 to $300 \mu \mathrm{as}$, or an average of $8 \%$ per object. More recently, Riess et al. (2014) and Casertano et al. (2016) measured two Cepheid parallaxes using HST Wide Field Camera 3 in scanning mode, with precision of 54 and $38 \mu$ as, respectively. With the addition of a few HIPPARCos measurements with errors of $300 \mu$ as, the resulting sample of 15 Galactic Cepheids had a weighted mean precision of $2.1 \%$ and provided one of three anchors for the determination of the Hubble constant (R16; see also Riess et al. 2011; Freedman et al. 2012). As discussed in Sect. 2, this precision requires the use of reddeninginsensitive Wesenheit magnitudes (Madore 1982) anchored in the near-infrared (NIR; R16, Macri et al. 2015), and a careful calibration of the characteristics of the $P-L$ relation.

In principle, TGAS parallaxes could provide a test of the zero point of the $P-L$ relation and thus contribute an independent calibration of the local distance scale (see Sect. 4); however, given the early nature of this release, we caution that systematic effects an order of magnitude below the typical measurement error, yet large enough to affect the resulting calibration, are difficult to rule out, thus the results of this analysis must be seen as tentative.

The rest of this paper is organized as follows. In Sect. 2 we describe the Cepheid sample and the underlying data on which the comparison is based. In Sect. 3 we carry out the test of the quality of TGAS parallaxes for stars in our sample. In Sect. 4 we provide a tentative analysis of the implications for the zero point calibration of the Leavitt Law and for the determination of the Hubble constant $H_{0}$. Finally, in Sect. 5 we summarize our conclusion and indicate the potential impact of the full-mission Gaia results on the determination of $H_{0}$.

\section{Data collected for the Cepheid parallax test}

The main sample we use to validate and interpret the Gaia TGAS parallax measurements is a set of 249 MW Cepheids identified by van Leeuwen et al. (2007) as having HIPPARCos measurements, together with the ground-based optical and near-infrared photometry included therein. We estimate the distance of these Cepheids on the basis of the infrared $P-L$ relation as calibrated by R16. In order to reduce the impact of object-by-object reddening and extinction, the $P-L$ relation is often formulated in terms of a so-called Wesenheit magnitude (Madore 1982; Macri et al. 2015, see R16 for details of our implementation). Wesenheit magnitudes are formed by subtracting from the primary magnitude a color term in the same direction as the reddening law; if the spectra are smooth and the reddening law is well constrained, a Wesenheit magnitude is then reddening-free, in the sense that a given source will have the same Wesenheit magnitude when exposed to different degrees of reddening. For Cepheids, Wesenheit magnitudes, especially those for which the primary filter is in the NIR, have the additional advantages that the color term is small and insensitive to the reddening law, the $P-L$ relation is insensitive to metallicity, and its intrinsic width is reduced. We use the same quantity as in R16:

$m_{H}^{W}=m_{160}-0.3861\left(m_{555}-m_{814}\right)$

where $m_{160}, m_{555}$, and $m_{814}$ are the Vega-system magnitudes in the WFC3 filters $F 160 W$ (NIR), F555W, and F814W, with central wavelength 1537,531 , and $802 \mathrm{~nm}$, respectively. The coefficient 0.3861 is appropriate to a Galactic reddening law (Fitzpatrick 1999) with $R=3.3$. Since the available photometry in van Leeuwen et al. (2007) is ground-based, while the best calibration of the $P-L$ relation obtained in R16 is based on HST data, we use the ground-to-HST transformations given in R16, Eqs. (10)-(12). The systematic uncertainty in this transformation, found by R16 to be $0.013 \mathrm{mag}$, is small compared to the 0.05 mag precision for the mean of the sample (see Sect. 3). However, to retain the full precision of future Gaia Cepheid parallax measurements, it will be crucial to measure the MW Cepheid mean magnitudes directly with HST, as discussed in Sect. 5. R16:

We adopt the primary calibration of the $P-L$ relation from

$M_{H}^{W}=-2.77-3.26 \log (P)$

where $M_{H}^{W}$ is the absolute magnitude in the same Wesenheit system of Eq. (1), and $P$ is the Cepheid period in days. The slope of this relation is constrained to within $0.02(0.6 \%)$ by 2500 Cepheids measured in over 20 different systems, including the Large Magellanic Cloud (LMC), M31, NGC 4258, and galaxies that hosted a Type Ia supernova. These Cepheids also demonstrate the lack of any significant break in slope near $P=$ $10 \mathrm{~d}$ which appears in optical bands (Ngeow \& Kanbur 2005; Ngeow et al. 2005).

The zero point of the $P-L$ relation was determined by R16 by using three independent geometric calibrations: trigonometric parallaxes for the 15 Galactic Cepheids mentioned in the Introduction, eight late-type detached eclipsing binaries in the LMC (Pietrzyński et al. 2013), and the geometric distance measurement based on the kinematic of a ring of $\mathrm{OH}$ masers in the galaxy NGC 4258 (Humphreys et al. 2013). Together, these measurements provide a calibration of the intercept in Eq. (2) with an uncertainty of $0.035 \mathrm{mag}$ ( $1.6 \%$ in distance). This calibration relates directly to the value of $H_{0}$; its role in our comparison will be discussed further in Sect. 3 .

The absolute magnitude of each Cepheid from Eq. (2), combined with its reddening-free apparent magnitude from Eq. (1), together provide a photometric parallax estimate for each individual Cepheid. The main statistical error in the photometric parallaxes is due to the intrinsic width of the $P-L$ relation, which Macri et al. (2015) estimate to be $\sim 0.08$ mag in the LMC 
for the $H$-band Wesenheit magnitude $M_{H}^{W}$. This width is measured empirically, and includes the intrinsic width of the instability strip, photometric errors, variations in reddening, and any other terms that might affect the ability to predict the absolute magnitude of a Cepheid from its period. Therefore the statistical uncertainty in each parallax estimate is $\sim 4 \%$ - substantially smaller than the median measurement error of $50 \%$ reported for the TGAS solution (see Sect. 3) - thus providing a suitable test of the quality of the TGAS parallaxes. However, these uncertainties will be very significant when the full mission results are available and the typical Gaia parallax uncertainty per star will likely be $<3 \%$. Systematic uncertainties, as in nearly all distance scale projections, will result primarily in a multiplicative scaling factor on the photometric parallax estimates, discussed further in Sects. 3 and 4.

\section{Parallax comparison}

As described in Sect. 2, the available magnitude and period information allows us to predict the parallax for the 212 stars in the van Leeuwen et al. (2007) list for which photometry is available in the $V, I, J$, and $H$ band and for which a TGAS parallax is available in DR1.

We carry out our analysis in parallax space, rather than in distance or photometry, because of the low signal-to-noise ratio ( $\mathrm{S} / \mathrm{N}$, median value $\sim 2$ ) of individual DR1 parallaxes. The determination of the absolute magnitude of a Cepheid follows from its apparent magnitude and parallax, where $M=m-\mu_{\pi}$ and $\mu_{\pi}$ is the distance modulus derived from parallaxes, including standard corrections for bias (often referred to as Lutz-Kelker bias) arising from the finite $\mathrm{S} / \mathrm{N}$ of parallax measurements (Lutz \& Kelker 1973; Hanson 1979). For $S / N<10$, the bias correction becomes large and complex, as one must contend with a skewing of the likelihood due to the selection bias (of presumably a disk population) which is asymmetric with distance and a non-Gaussian conversion from parallax to magnitude. A number of papers have argued about the optimal way to contend with these issues for low S/N parallax measurements (Francis 2014; Feast 2002; Hanson 1979; Benedict et al. 2007). The approach we have adopted here is to use the high precision of Cepheid magnitudes and $P-L$ parameters $(S / N \sim 100)$ in $\mathrm{R} 16$ to predict the parallaxes to the Gaia DR1 parallaxes and compare the results. In essence, we are using the Gaia first release catalogue as a test of R16, rather than to derive a completely independent calibration of the Cepheid $P-L$. We expect the best use of the Gaia results to change dramatically as the Gaia precision improves by more than an order of magnitude.

The distribution of TGAS vs. photometric parallaxes for the 212 Cepheids is shown in Fig. 1, with the error bars indicating the reported TGAS uncertainty. Clearly the distributions are in good basic agreement, with the exception of the outlier RW Cam (labeled in Fig. 1), which has a TGAS parallax of $3.687 \pm 0.797$ mas, vs. a predicted value of 0.608 mas. RW Cam is known to have a very luminous, B8.2 III companion (Evans \& Udalski 1994), instead of the typical main-sequence companion (Bohm-Vitense \& Proffitt 1985); Fernie (2000) concludes that the bright companion is the cause of its unusual photometric properties. The bright companion can affect both the photometric distance determination and the parallax measurement (see, e.g., the discussion in Anderson et al. 2016). Therefore we exclude RW Cam from further analysis.

As discussed in Sect. 2, we estimate the uncertainty in the distance modulus estimate of each Cepheid in $0.08 \mathrm{mag}$ (Macri et al. 2015); this includes both the intrinsic width of

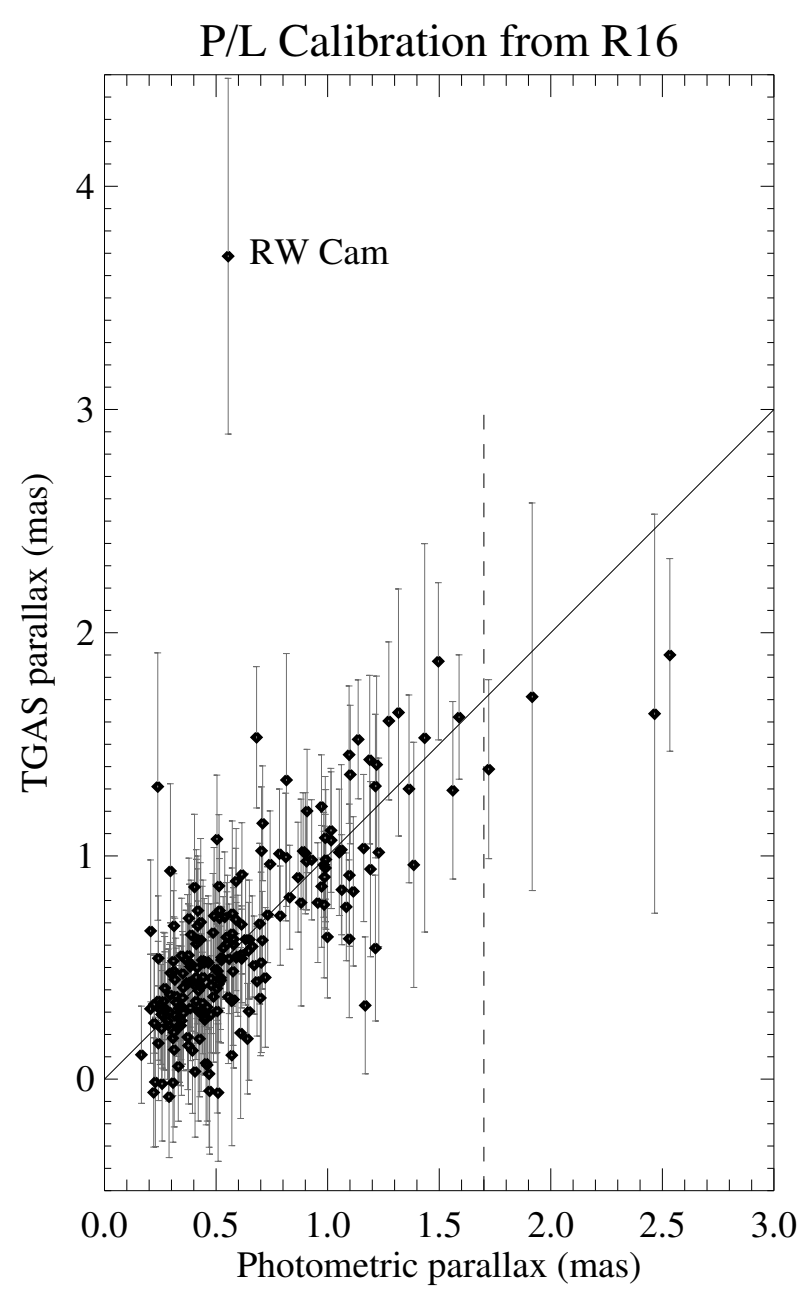

Fig. 1. TGAS parallaxes vs. photometric parallax estimates. The error bars are the formal errors as reported in DR1. Formal uncertainties in photometric parallax estimates are small, about 5\%. As noted in the text, the outlier RW Cam is likely to have a bright companion. Note the apparent systematic offset for stars with large photometric parallaxes; such stars also have larger nominal errors.

the $P-L$ relation, which affects the absolute magnitude estimated from the period, and the photometric errors, which enter in the distance modulus calculation. As the width of the relation is empirically determined, any other terms that might affect the ability to predict distances for individual Cepheids are also included in this value. Figure 2 shows the ratio of photometric to TGAS parallax errors for the 211 stars remaining in our sample; the photometric errors are typically 13 times smaller than the TGAS errors (median ratio 0.072), and in all cases at least 4 times smaller. Therefore the parallax test is dominated by the TGAS errors, making this a very valuable sample to carry out the comparison.

With the exception of RW Cam, most points in Fig. 1 lie within the nominal $1 \sigma$ error bars, suggesting that perhaps the errors may be overestimated. Indeed, under the simplistic model that the photometric and TGAS parallax are equal aside from measurement errors, the $\chi^{2}$ per degree of freedom (no free parameters) is 0.63 (a less than 1 in $10^{5}$ chance for a pure Gaussian distribution with the correct error estimate). If we assume that the errors are underestimated by a constant multiplicative factor, its likely value is $1-\sqrt{0.63}$, or about $20 \%$. While the assumption of a single multiplicative factor may be simplistic, the conclusion that the error are underestimated - at least for the sample of 


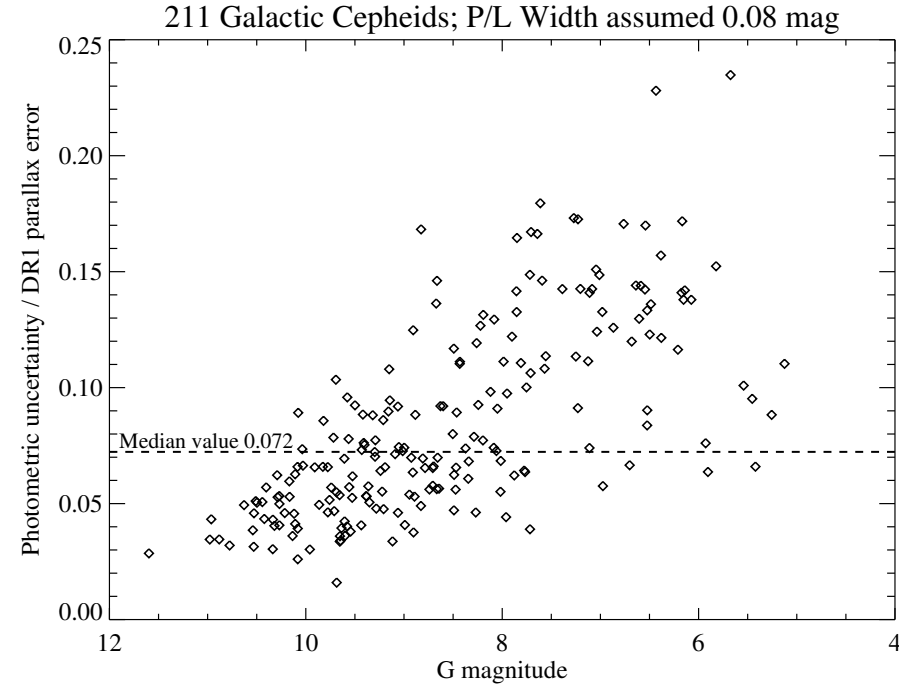

Fig. 2. Ratio of the uncertainty in the photometric parallaxes to the TGAS catalog errors for Cepheids in our sample, as a function of apparent $G$ magnitude. The median value of the ratio is 0.072 , and the maximum is 0.24 , indicating that the photometric errors are negligible in comparison to the TGAS measurement error. The photometric errors are based on an assumed width of $P / L$ relation of 0.08 mag, which combines its intrinsic width with the uncertainties in ground-based magnitudes. The strong correlation is a distance effect: the brighter Cepheids are typically closer, so that a given fractional error in distance (deriving from a constant error in distance modulus) translates to a larger uncertainty in the photometric parallax.

Cepheids for which we carry out the comparison - appears solid, as there is no commonality of information between our photometric parallax estimates and the TGAS parallax measurements. Lindegren et al. (2016) indicate that the formal uncertainties reported by the solution process have been inflated by a factor $F$ derived from a comparison with HIPPARCos parallaxes (see their Eq. (4) and Appendix B; $F$ has a minimum value of 1.4). Our comparison suggests that, at least for the stars we consider, this inflation factor may have been overestimated. Lindegren (priv. comm.) has suggested that the inflation factor may be larger for stars of extreme colors and smaller for those near the middle of the color distribution, which includes the majority of our targets; if this is the case, errors may be systematically overestimated for Cepheids, but not for stars with extreme colors. In the following we consider two options: the errors as reported, and scaled errors that are 0.8 times the values reported.

In addition to the inflation factor applied to the formal errors, Lindegren et al. (2016) also indicate that there may be an additional systematic error of 0.3 mas on the reported parallaxes, and that parallax errors may be correlated on scales up to $10^{\circ}$. We tested for the possibility of a correlation by analyzing the residuals with respect to the photometric parallax estimates. The Cepheids fainter than $G=6 \mathrm{mag}$ in our sample form 20301 unique pairs, of which $0.5 \%$ are separated by $<2^{\circ}$, and $6.7 \%$ by $<10^{\circ}$. (Parallaxes for brighter stars may be systematically underestimated, as discussed below.) Therefore our Cepheids are very weakly correlated according to the Lindegren et al. (2016) criteria. These relatively close pairs offer the opportunity to estimate the angular correlation of parallaxes directly from the data. We find that the two-point correlation between residuals in the 1363 unique pairs separated by $<10^{\circ}$ is $19 \pm 34 \mu$ as, suggesting that the correlation on such scales is too small to be detected with this test. Note that our test treats

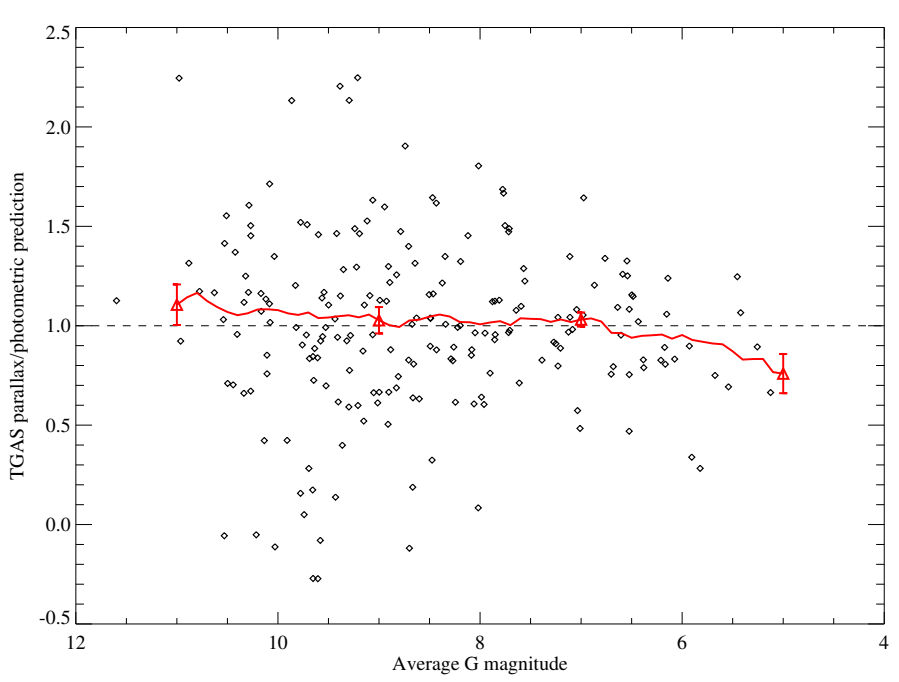

Fig. 3. Ratio between TGAS and photometric parallaxes as a function of average $\mathrm{G}$ magnitude as given in the DR1 data. The red symbols show the mean and the error in the mean (based on the actual dispersion, not the nominal errors) for bins 2 mag wide; the red line shows the mean ratio in moving bins 2 mag wide centered at the magnitude in the abscissa. Note that three stars exceed the range of the $Y$ axis and are not shown, but are included in the averages. This comparison suggests that TGAS parallaxes for very bright stars $(G<6)$, for which the central pixel will typically be saturated even at the shortest gating used, may be systematically underestimated.

all separations smaller than $10^{\circ}$ equally, and is therefore insensitive to a correlation that changes sign within that range (e.g., as suggested by Fig. D3 in Lindegren et al. 2016). Nonetheless, our finding of smaller errors than reported suggests that additional systematics and correlations, if they exist, are significantly smaller than the formal uncertainties.

The distribution shown in Fig. 1 also suggests that for some of the largest photometric parallaxes ( $>1.7$ mas), the TGAS parallaxes may be consistently low. These stars are also among the brightest in the sample. All of the Cepheids in our sample are brighter than the Gaia saturation limit ( $\sim 12 \mathrm{mag}$ in the Gaia $G$ band) and therefore require the use of gating (Gaia Collaboration 2016b; Fabricius et al. 2016; Lindegren et al. 2016; Gaia Collaboration 2016a) to be measured; the details of the process depend on the brightness of each individual star. In Fig. 3 we show the ratio of measured to predicted parallax as a function of average $G$ magnitude as measured by Gaia; the red symbols show the average ratio in bins 2 mag wide. For stars fainter than $G=6 \mathrm{mag}$, the unweighted average ratio between TGAS and predicted parallax is $1.03 \pm 0.04$ (uncertainty in the mean); for the 9 stars brighter than $G=$ $6 \mathrm{mag}$, the average ratio is $0.86 \pm 0.06$. A formal significance test indicates a significant difference but is of limited value, as the error distribution is not fully characterized (more on this below); however, stars brighter than $G \sim 6$ are in fact treated slightly differently from fainter stars. At $G=6$, a larger download window is used (Fabricius et al. 2016); near this magnitude, the core of the stellar image will likely saturate at the shortest gating interval used (TDI gate 4, 16 lines; Gaia Collaboration 2016b). Thus a separation between stars brighter and fainter than $G \sim 6 \mathrm{mag}$ is naturally driven by the specifics of the measurement process and merits the a priori check. Additional confirmation that the difference is likely in the TGAS measurements can be obtained by considering the 3 stars for which $G<6$ and an independent trigonometric parallax measurement has been obtained with 


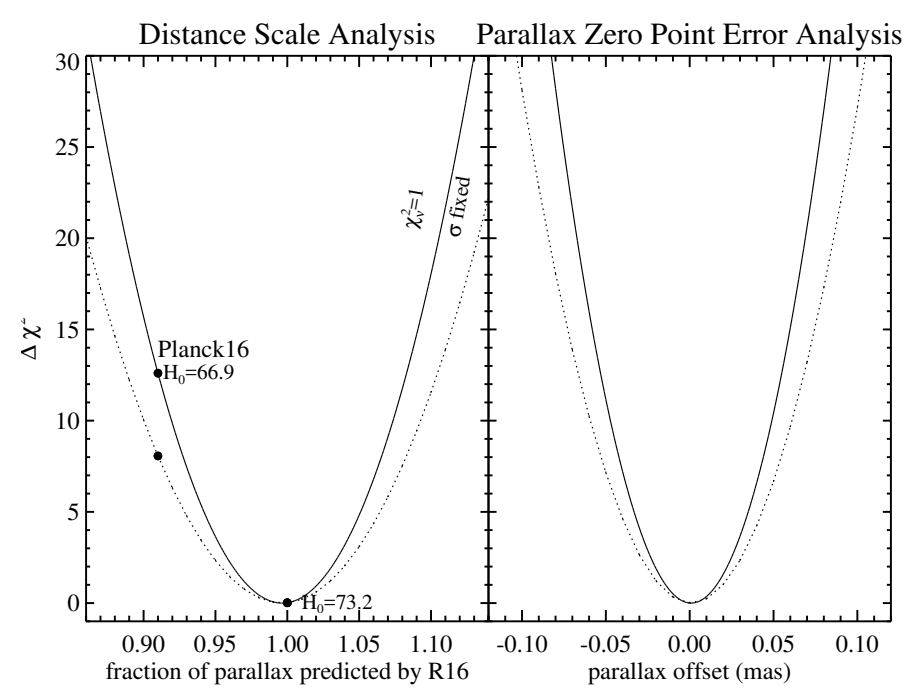

Fig. 4. Comparing Gaia and predicted parallaxes to test either the distance scale or the parallax zero point. Shown is the change in $\chi^{2}$ caused by a multiplicative term in the photometric parallaxes (left panel) or an additive term in DR1 parallaxes (right panel). In each panel, the dashed curve uses the nominal errors, while the solid curve is for errors scaled by a factor 0.80 , as suggested in order to obtain $\chi^{2}=1$ per degree of freedom. See text for more discussion.

HST (Benedict et al. 2002, 2007; van Leeuwen et al. 2007). For these, the Gaia DR1 values are also low, with a mean which is $0.70 \pm 0.35$ mas smaller than the independent parallaxes, indicating that the apparent difference between TGAS and photometric parallaxes for $G<6$ is not due to any issues with photometric parallax estimates for nearby, bright stars. To be conservative, we exclude the 9 stars brighter than $G=6$ mag from further analysis; their exclusion does not change significantly the value of the reduced $\chi^{2}$ or the apparent error overestimate. We also note that some of these Cepheids, e.g., SU Cas and BG Cru, have been sometimes classified as First Overtone instead of Fundamental Mode Cepheids, which might contribute to their anomalous parallax; therefore we consider the parallax underestimate at $G<6 \mathrm{mag}$ as tentative.

There are thus 202 Cepheids from van Leeuwen et al. (2007) with parallaxes in the TGAS sample that provide the means to explore the present distance scale in R16 or alternatively the possibility of a zeropoint parallax error in Gaia. Despite the one outlier and the possible underestimation of parallaxes for the few Cepheids brighter than $G=6 \mathrm{mag}$, the agreement between the TGAS parallaxes and the photometric distances predicted from the R16 Cepheid calibration is remarkably good. This is consistent with the comparison in Lindegren et al. (2016), who use 141 stars in the Fouqué et al. (2007) sample, likewise excluding the nearest Cepheids (within $1 \mathrm{kpc}$ ).

In Fig. 4 we show the change in $\chi^{2}$ that would result from either a multiplicative term in the photometric parallaxes (left panel) or an additive term in the TGAS parallaxes (right panel). The formal minimum for the multiplicative term is obtained for a value of $0.997 \pm 0.025$ with 0.8 scaled errors and $0.997 \pm 0.031$ for nominal errors, consistent with no difference. For a global additive term, we find a preferred value of $1 \mu$ as, with a formal uncertainty of $15 \mu$ as for scaled errors (19 $\mu$ as for nominal errors). An additive term could arise, e.g., from a parallax zero point error due to from imperfections in the correction for Basic Angle variations (Michalik \& Lindegren 2016). Lindegren et al. (2016) carry out several tests for zero point errors, finding terms with magnitude $\sim 0.1$ mas and possible dependence on hemisphere and color; given the small number of sources in our sample, we limit our analysis to a single global zero point offset, which we find to be less than $20 \mu$ as $(1 \sigma)$.

Note that in our analysis, the assumption is implicitly made that the term in consideration (additive or multiplicative) is the only systematic in the data. Additional systematics could exist (e.g., terms that depend on magnitude, color, phase of observations, or location in the sky), and if they are below the measurement errors, would not be possible to detect without a specific test, but could reduce the precision with which an additive or multiplicative term can be determined.

We conclude that the TGAS parallaxes for the sample of Galactic Cepheids under consideration are in very good agreement, to the limit of their current precision, with the predicted parallaxes based on the calibration of R16. There is an indication and independent confirmation of an underestimation of parallax for the few stars brighter than $G=6 \mathrm{mag}$, and we estimate that the reported errors appear conservatively overestimated by approximately $20 \%$.

\section{Implications for the local value of Hubble constant}

The multiplicative scaling of the parallax predicted from Cepheid photometry is formally equivalent to a change in the zero point of the calibration of the Leavitt Law; from the analysis in R16, this is equivalent to a change in the estimated value of $H_{0}$. The R16 value of $73.2 \mathrm{~km} \mathrm{~s}^{-1} \mathrm{Mpc}^{-1}$ corresponds of course to a multiplicative factor of 1.0 , which is well within the $1 \sigma$ range of the values reported in the previous section. The bestfit factor of $0.997 \pm 0.025( \pm 0.031$ without rescaling the errors) would produce a "recalibration" to $H_{0}=73.0 \mathrm{~km} \mathrm{~s}^{-1} \mathrm{Mpc}^{-1}$ if interpreted as such. The Planck Collaboration Int. XLVI (2016) value of $66.9 \mathrm{~km} \mathrm{~s}^{-1} \mathrm{Mpc}^{-1}$, based on Planck cosmic microwave background (CMB) data with $\Lambda$ cold dark matter (CDM), corresponds to a factor of 0.91 , which would produce a change in total $\chi^{2}$ of 8.1 with the nominal errors, or of 12.6 if the errors are scaled by a factor 0.8 , and thus a tension at the $2.5-3.5 \sigma$ level, respectively, with the DR1 parallaxes. Note that this discrepancy is much larger than the differences between any two sets of anchors in R16. However, it is difficult to determine whether there are additional systematic uncertainties globally affecting this analysis. Specifically, we do not know if we can reasonably combine so many low $\mathrm{S} / \mathrm{N}$ measurements to produce one, high $\mathrm{S} / \mathrm{N}$ measurement without penalty, and thus the present result is tentative.

\section{Discussion}

The Gaia mission appears to be off to a tremendous start, and there is little doubt that the full mission will produce results of great import for cosmology. An analysis of the DR1 parallaxes for just over 200 Galactic Cepheids with accurate photometric distances shows that (1) the quality of the parallaxes is extremely good, with probably a slight $(20 \%)$ overestimate of the uncertainties; and (2) even at DR1 precision, the parallaxes are consistent with the local value of $H_{0}$ as determined by R16, and mildly inconsistent at the level of $2.5 \sigma(3.5 \sigma$ if the errors are rescaled) with the smaller value of $H_{0}$ inferred from the CMB with $\Lambda$ CDM. This comparison will be greatly sharpened when the DR2 results become available, in about one year.

While individual parallaxes have been measured for a small number of Cepheids at better precision than Gaia DR1, the new 
and exciting feature of DR1 is the angular breadth of the measurements, providing for $\sim 300 \mu$ as precision for hundreds of classical Cepheids. The dispersion of $V, I$ and $H$-band based Wesenheit magnitudes and log periods has been observed to be 0.08 magnitudes for over 1000 objects in the LMC (Macri et al. 2015), which would result in random, individual errors of just $\sim 20 \mu$ as. The fact that the $\chi_{v}^{2}=0.63(N=202)$ likely results from a $20 \%$ overestimate of Gaia DR1 errors, as the errors in the predictions are too small to have any impact.

The sample mean of the DR1 parallaxes for the Cepheids we consider has a nominal error of $\sim 20 \mu$ as. If there are no systematic errors at this level in the DR1 measurements, then these Cepheids would produce an independent calibration of the Cepheid distance scale with an uncertainty of $3.1 \%$ (original errors) to $2.5 \%$ ( $80 \%$ errors), competitive with the best geometric calibrations from NGC 4258 (2.6\%), previous MW Cepheid parallaxes $(2.1 \%)$ and detached eclipsing binaries in the LMC (2.0\%) (R16). The factor of twenty or more improvement expected for Gaia parallaxes by mission end will push the uncertainty due to geometric distance calibration well below $1 \%$, assuming systematics can be kept under comparable control.

However, the geometric calibration of Cepheids, central to measuring the Hubble constant, depends not on just the mean parallax precision of the sample but also on the ability to compare them photometrically to their cousins in distant galaxies. The photometry of extragalactic Cepheids in SN Ia hosts can only be measured at present in space with HST and has been achieved most extensively with WFC3. On the other hand, the photometry of the Gaia DR1 Cepheid sample analyzed here was obtained from various ground-based sources. Due to the high foreground extinction of the MW fields and in external galaxies, the use of NIR magnitudes and colors is especially important. Ground-based NIR filter systems are based on natural (and nightly changing) breaks in water and $\mathrm{OH}$ emission and do not well match the space based system. This produces systematic uncertainties at the level of approximately $0.02 \mathrm{mag}$, including the relative uncertainties in NIR zeropoints and dereddened colors (R16). These uncertainties are currently below the precision of the geometric calibration of the distance scale, but will be well above the precision that can be achieved with Gaia full-mission results.

One of the best ways to mitigate the systematic error resulting from comparing ground and space-based Cepheid photometry is to observe the MW Cepheids with HST's WFC3. We have undertaken a series of HST programs to measure the magnitudes of $\sim 50$ MW Cepheids with relatively low extinction and we have employed rapid spatial scanning with HST to avoid the saturation which would occur with direct imaging of such bright stars. In the future, the combination of these 50 parallaxes from Gaia and their HST photometry in F555W, F814W, and F160W should produce a complete and effective calibration of extragalactic Cepheids with a mean error of $\sim 0.5 \%$, and an anchor for a $1 \%$ determination of the Hubble constant.

We have chosen to use the Gaia DR1 parallaxes as a test rather than an augmentation of the current calibration of $H_{0}$ by R16 to avoid the complication of Lutz-Kelker type bias corrections that would be large and necessary for parallax measurements with mean $S / N \sim 2$, and in recognition that these parallaxes are expected to dramatically improve in the near term (thus reducing the need for such corrections as well).

This is the start of an exciting phase of measurement and perhaps discovery in the long-lived field of parallax measurement, with the best yet to come.

Acknowledgements. This work has made use of data from the European Space Agency (ESA) mission Gaia (http://www.cosmos.esa.int/gaia), processed by the Gaia Data Processing and Analysis Consortium (DPAC, http://www. cosmos.esa.int/web/gaia/dpac/consortium). Funding for the DPAC has been provided by national institutions, in particular the institutions participating in the Gaia Multilateral Agreement. Support for this work was provided by NASA through program GO-13101 from the Space Telescope Science Institute, which is operated by AURA, Inc., under NASA contract NAS 526555, and by the Agenzia Spaziale Italiana (ASI) through ASI grant 2014-025R.1.2015. We also thank an anonymous referee for pointing out areas that needed further clarification in the original manuscript, and Dr. Lindegren for illuminating discussions on the error estimates in DR1.

\section{References}

Anderson, R. I., Casertano, S., Riess, A. G., et al. 2016, ApJS, 226, 18 Benedict, G. F., McArthur, B. E., Fredrick, L. W., et al. 2002, AJ, 123, 473 Benedict, G. F., McArthur, B. E., Feast, M. W., et al. 2007, AJ, 133, 1810 Bohm-Vitense, E., \& Proffitt, C. 1985, ApJ, 296, 175

Casertano, S., Riess, A. G., Anderson, J., et al. 2016, ApJ, 825, 11

Evans, N. R., \& Udalski, A. 1994, AJ, 108, 653

Fabricius, C., Bastian, U., Portell, J., et al. 2016, A\&A, 595, A3

Feast, M. 2002, MNRAS, 337, 1035

Fernie, J. D. 2000, AJ, 120, 978

Fitzpatrick, E. L. 1999, PASP, 111, 63

Fouqué, P., Arriagada, P., Storm, J., et al. 2007, A\&A, 476, 73

Francis, C. 2014, MNRAS, 444, L6

Freedman, W. L., Madore, B. F., Scowcroft, V., et al. 2012, ApJ, 758, 24

Gaia Collaboration (Brown, A. G. A., et al.) 2016a, A\&A, 595, A2

Gaia Collaboration (Prusti, T., et al.) 2016b, A\&A, 595, A1

Hanson, R. B. 1979, MNRAS, 186, 875

Høg, E., Fabricius, C., Makarov, V. V., et al. 2000, A\&A, 355, L27

Humphreys, E. M. L., Reid, M. J., Moran, J. M., Greenhill, L. J., \& Argon, A. L. 2013, ApJ, 775, 13

Leavitt, H. S., \& Pickering, E. C. 1912, Harvard College Observatory Circular, 173,1

Lindegren, L., Lammers, U., Bastian, U., et al. 2016, A\&A, 595, A4

Lutz, T. E., \& Kelker, D. H. 1973, PASP, 85, 573

Macri, L. M., Ngeow, C.-C., Kanbur, S. M., Mahzooni, S., \& Smitka, M. T. 2015 , AJ, 149, 117

Madore, B. F. 1982, ApJ, 253, 575

Michalik, D., \& Lindegren, L. 2016, A\&A, 586, A26

Michalik, D., Lindegren, L., \& Hobbs, D. 2015, A\&A, 574, A115

Mora, A., Biermann, M., Brown, A. G. A., et al. 2014, in Space Telescopes and Instrumentation 2014: Optical, Infrared, and Millimeter Wave, Proc. SPIE, 9143, 91430

Ngeow, C.-C., \& Kanbur, S. M. 2005, MNRAS, 360, 1033

Ngeow, C.-C., Kanbur, S. M., Nikolaev, S., et al. 2005, MNRAS, 363, 831

Perryman, M. A. C., Lindegren, L., Kovalevsky, J., et al. 1997, A\&A, 323, L49 Pietrzyński, G., Graczyk, D., Gieren, W., et al. 2013, Nature, 495, 76

Planck Collaboration Int. XLVI. 2016, A\&A, 596, A107

Prusti, T. 2012, Astron. Nachr., 333, 453

Riess, A. G., Macri, L., Casertano, S., et al. 2011, ApJ, 730, 119

Riess, A. G., Casertano, S., Anderson, J., MacKenty, J., \& Filippenko, A. V. 2014, ApJ, 785, 161

Riess, A. G., Macri, L. M., Hoffmann, S. L., et al. 2016, ApJ, 826, 56

van Leeuwen, F. 2007, in Hipparcos, the New Reduction of the Raw Data, Astrophys. Space Sci. Lib., 350

van Leeuwen, F., Feast, M. W., Whitelock, P. A., \& Laney, C. D. 2007, MNRAS, 379,723 